\title{
Outcome of Laser Photocoagulation for Retinopathy of Prematurity
}

\author{
Kiran Balagopal ${ }^{1}$, Parvathy Reghudev ${ }^{2}$ \\ 1,2 Department of Ophthalmology, Travancore Medical College, Kollam, Kerala, India.
}

\section{ABSTRACT}

\section{BACKGROUND}

Retinopathy of prematurity (ROP) is a disease affecting the retina of premature infants due to retinal neovascularization and the spectrum of outcome findings in ROP ranges from the most minimal sequelae without affecting vision in the mild cases to bilateral, irreversible, and total blindness in more severe cases.In India children less than $1500 \mathrm{~g}$ have almost 38 percent chance of developing any stage of ROP and 7 - $16 \%$ chance of developing threshold ROP or blinding ROP. (2)Laser photocoagulation has replaced cryotherapy as the established treatment for ROP in most centers and this study aims to evaluate the functional and structural outcome in preterm infants who received laser photocoagulation for retinopathy of prematurity. In this study we wanted to find out the structural outcome of retinopathy of prematurity infants who underwent laser photocoagulation. We also wanted to find out the functional outcome of ROP preterm infants who underwent laser photocoagulation and identify the factors affecting the structural outcome in retinopathy of prematurity.

\section{METHODS}

A hospital based descriptive study was conducted among 31 preterm infants with retinopathy of prematurity who received laser photocoagulation treatment after obtaining parental consent in the Retina clinic, Regional Institute of Ophthalmology, Trivandrum. Screening examination was done at 34 weeks or 4 weeks postnatal whichever was earlier. Those infants who were detected to have ROP and were given laser photocoagulation by the ophthalmologist were followed up till retina becomes stable / ROP regresses. Thereafter follow up examinations were performed at 3 months, 6 months and 10 months. Structural outcome was classified as favorable and unfavorable. Visual or functional outcome was assessed by noting the fixation pattern, ocular alignment for strabismus and by performing cycloplegic refraction (retinoscopy) to rule out refractive errors. Data was entered into excel sheet and statistical analysis was done using SPSS trial version.

\section{RESULTS}

The study was conducted in 31 preterm infants with Retinopathy of Prematurity who underwent laser photocoagulation in Regional Institute of Ophthalmology Trivandrum. The structural and functional outcome was assessed at 3months, 6 months and 10 months. Of the 62 eyes of 31 infants, stage 2 and 3 comprised $48.4 \%$ each.3.2\% of eyes had stage 1 disease affecting zone1.None had stage 4 or $5.0 f$ the 62 eyes, majority had zone 2 disease $(83.9 \%)$. Only 10 eyes had zone1 disease. Out of the 60 eyes, 54 eyes had a favorable structural outcome at 3 months.The functional outcome was assessed in terms of visual behavior by assessing the fixation of light, whether child is following light and by preferential looking pattern. Out of the 30 babies only 1 had an unfavorable visual behavior. The baby was not fixing and following light. Out of the 30 infants, 2 had (6.6\%) strabismus at 6 months and 10 months. Myopia was the predominant refractive error in the studied group accounting for about $55 \%$. Among the study group $16.7 \%$ had hypermetropia.

\section{CONCLUSIONS}

The incidence of favorable structural outcome in our study was 90\%.Low gestational age and low birth weight were associated with an unfavorable structural outcome. There is a high prevalence of refractive errors in our study group accounting for about $71.5 \%$ at 6 months. The refractive errors increased at 10 month follow up to $77 \%$. Among the refractive errors, myopia predominates accounting 55\% at 6 months and $61 \%$ at 10 months.

\section{KEY WORDS}

Laser Photocoagulation, Retinopathy of Prematurity
Corresponding Author: Dr. Parvathy Reghudev, Assistant Professor, Department of Ophthalmology, Travancore Medical College, Kollam, Kerala, India.

E-mail: parvathyrdev@gmail.com

DOI: 10.14260/jemds/2022/46

How to Cite This Article:

Balagopal K, Reghudev P. Outcome of laser photocoagulation for retinopathy of prematurity. J Evolution Med Dent Sci 2022;11(01):241-245, $10.14260 /$ jemds $/ 2022 / 46$

Submission 29-12-2021,

Peer Review 05-01-2022,

Acceptance 29-01-2022,

Published 31-01-2022.

Copyright (C) 2022 Kiran Balagopal et al. This is an open access article distributed under Creative Commons Attribution License [Attribution 4.0 International (CC BY 4.0)] 


\section{BACKGROUND}

Retinopathy of prematurity is a disease affecting the retina of premature infants. Its key pathological change, retinal neovascularization appears to be associated with local ischemia and subsequent development of neovascularization. The spectrum of outcome findings in ROP ranges from the most minimal sequel without affecting vision in the mild cases to bilateral, irreversible and total blindness in more severe cases. The retinopathy was first reported by Terry in 1942 and suggested that this disorder, retrolental fibroplasia appeared to develop after birth since several babies who manifested the disease had normal examinations shortly after birth. ${ }^{1}$

Retinopathy of prematurity (ROP) is a leading cause of blindness of children in areas of the world that provide neonatal intensive care services to premature newborns. Among the preventable causes of childhood blindness (57\%, ROP figures very high. ${ }^{2}$ Furthermore, developing countries are witnessing an increase in retinopathy of prematurity (ROP); therefore, ROP has now become a leading cause of childhood blindness worldwide. Asians also have an increased risk of ROP. In India, the incidence of ROP is estimated to be about $24 \%$ and in children less than 1500 gal most 38 percent have chance of developing any stage of ROP and $7-16 \%$ chance of developing threshold ROP or blindingROP. 3

National Cooperative Study of Oxygen and ROP in the mid $1950 \mathrm{~s}^{4}$ established the link between excessive Oxygen therapy and ROP which led to severe restriction of oxygen administration in premature babies which in turn resulted in increased mortality. This observation led to the more liberal use of oxygen and a resurgence of ROP known as the second epidemic.

No standard guide lines existed for the treatment of ROP before the multicenter trial Cryotherapy for Retinopathy of Prematurity Study (CRYO-ROP Study) which established that the treatment with cryotherapy for threshold ROP reduced the incidence of unfavorable outcome. Laser photocoagulation has replaced cryotherapy as the established treatment for ROP in most centres. The guidelines for treatment changed after the early treatment of Retinopathy of Prematurity Study (ETROP Study) and the follow up reported a better structural outcome. Cryotherapy to the avascular anterior retina in ROP eyes with threshold disease has been demonstrated to reduce by approximately half the incidence of an unfavorable outcome such as macular dragging, retinal detachment, or retrolental cicatrix formation. The treatment of choice for ROP has long shifted from cryotherapy to peripheral diode laser photocoagulation soon after clinical studies showed that laser therapy is superior to cryotherapy. Most ophthalmologists prefer laser therapy to retinal cryoablation because of its superior clinical efficacy and costeffectivity. ${ }^{5,6}$

This study aims to evaluate the functional and structural outcome in preterm infants who received laser photocoagulation for retinopathy of prematurity in RIO Trivandrum.

In this study we wanted to find out the structural outcome of retinopathy of prematurity infants who underwent laser photocoagulation. We also wanted to find out the functional outcome of ROP preterm infants who underwent laser photocoagulation and identify the factors affecting the structural outcome in retinopathy of prematurity.

\section{METHODS}

A hospital based descriptive study was conducted among 24 preterm infants with retinopathy of prematurity who received laser photocoagulation treatment after obtaining parental consent in the Retina clinic, Regional Institute of Ophthalmology, Trivandrum. Screening examination was done at 34 weeks or 4 weeks postnatal whichever was earlier. Those infants who were detected to have ROP and were given laser photocoagulation by the ophthalmologist were followed up till retina becomes stable / ROP regresses. Thereafter follow up examinations were performed at 3 months, 6 months and 10 months. Structural outcome was classified as favourable and unfavourable. Visual or functional outcome was assessed by noting the fixation pattern, ocular alignment for strabismus and by performing cycloplegic refraction (retinoscopy) to rule out refractive errors. Data was entered into excel sheet and statistical analysis was done using SPSS trial version.

\section{RESULTS}

A total of 31 infants (62 eyes) were enrolled into the study over a period of one year. Among these 31 , one baby was not brought for follow up and was dropped from the study. Thus a total of 30 infants were studied and followed up for a period of 10 months. Among them 16 (51.6\%) were male and 15 $(48.4 \%)$ were females.

\begin{tabular}{|c|c|c|c|}
\hline Factors & Category & Frequency & Percentage (\%) \\
\hline \multirow{4}{*}{$\begin{array}{l}\text { Gestational Age of the } \\
\text { study participants }\end{array}$} & $<28$ weeks & 2 & $6.5 \%$ \\
\hline & $28-30$ weeks & 12 & $38.7 \%$ \\
\hline & 30-32 weeks & 9 & $29 \%$ \\
\hline & 32-34weeks & 8 & $25.8 \%$ \\
\hline \multirow{4}{*}{$\begin{array}{c}\text { Post conceptional age } \\
\text { of diagnosis }\end{array}$} & $30-32$ weeks & 1 & $3.2 \%$ \\
\hline & $32-34$ weeks & 13 & $41.9 \%$ \\
\hline & 34-36 weeks & 8 & $25.8 \%$ \\
\hline & $36-38$ weeks & 9 & $29 \%$ \\
\hline \multirow{4}{*}{$\begin{array}{l}\text { Post-conceptional age } \\
\text { at which treatment } \\
\text { started }\end{array}$} & 30-32 weeks & 1 & $3.2 \%$ \\
\hline & 32-34 weeks & 9 & $29 \%$ \\
\hline & 34-36 weeks & 8 & $25.8 \%$ \\
\hline & $36-38$ weeks & 13 & $41.9 \%$ \\
\hline \multicolumn{2}{|c|}{ Total } & 31 & 100 \\
\hline
\end{tabular}

Of the 31 babies majority belonged to $28-30$ weeks gestational age (38.7\%). The gestational age ranged from 26 weeks to 38 weeks. The mean gestational age was 30.2 weeks (SD 1.8weeks). Among the babies majority was in $<1.5$ kgcategory $(80.6 \%)$. The birth weight ranged from a minimum of $850 \mathrm{gm}$ to maximum of $1.88 \mathrm{~kg}$. The mean birth weight was $1.24 \mathrm{~kg}$ (SD $0.26 \mathrm{~kg}$ ).

Majority of infants were diagnosed to have ROP at a post conceptional age (PCA) of 32-34weeks.Age of detection of ROP ranged from as early as 30 weeks to as late as 38 weeks.

Most of the babies underwent laser photocoagulation after 34 weeks since most of the babies detected with ROP 
developed threshold disease after 34 weeks of post conceptional age. All the infants had history of respiratory distress at birth (RDS) and all of them had neonatal ICU admission. All babies were given supplemental oxygen. $3 \%$ of the infants had neurological disorder like seizure or intracranial hemorrhage. 54.8\%had neonatal sepsis.

\begin{tabular}{|ccc|}
\hline Stages of ROP & Frequency & Percentage (\%) \\
Stage 1 & 2 & $3.2 \%$ \\
Stage 2 & 30 & $48.4 \%$ \\
Stage 3 & 30 & $48.4 \%$ \\
Total & $\mathbf{6 2}$ & $\mathbf{1 0 0 \%}$ \\
\hline \multicolumn{3}{|c|}{ Table 2. Distribution of Retinopathy of Prematurity stages among the } \\
study participants (n-62)
\end{tabular}

Of the 62 eyes of 31 infants, stage 2 and 3 comprised $48.4 \%$ each.3.2\% of eyes had stage 1 disease affecting zone1. None had stage 4 or 5 . Of the 62 eyes; majority had zone 2 disease (83.9\%). Only 10 eyes had zone 1 disease.Of the 62 eyes, $72.5 \%$ had more than 6 clock hours of involvement. Out of the 62 eyes, 53eyes had plus disease.

\section{Analysis of Outcome of Laser Photocoagulation}

In this study screening examination is done at 34 weeks or 4 weeks postnatal whichever is earlier. Those infants who are detected to have threshold or prethreshold type AROP were given laser photocoagulation using Laser Indirect Ophthalmoscopic delivery system by ophthalmologists and followed up till retina becomes stable or ROP regresses. Thereafter follow up examinations are performed at 3 months, 6 months and 10 months. Structural outcome was considered unfavourable ${ }^{7}$ if there is (1) a posterior retinal fold involving the macula/macular drag (2) a retinal detachment involving the macula, or (3) retrolental tissue or "mass" obscuring the view of the posterior pole. In the present study the findings are:

\begin{tabular}{|ccc|}
\hline Structural Outcome & Frequency & Percentage (100\%) \\
Favourable & 54 & $90.0 \%$ \\
Unfavourable & 6 & $10.0 \%$ \\
Total & $\mathbf{6 0}$ & $\mathbf{1 0 0 . 0 \%}$ \\
\hline \multicolumn{2}{|c|}{ Table 3. Distribution of structural outcome of retina on study } \\
participants at 3 months (n-60)
\end{tabular}

Out of the 60 eyes, 54 eyes had a favourable outcome at 3 months. There was no structural sequel like vessel straightening, retrolental fold, foveal ectopia, macular drag, retinal detachment, retinal thinning or disc pallor. 6 eyes had an unfavorable structural outcome (10\%). All 6 eyes had macular drag. The retinal findings remained stable at 6 months and ten months of follow up in all these infants.

\begin{tabular}{|cccccccc|}
\hline \multirow{2}{*}{ Factors } & Category & $\begin{array}{c}\text { Favourable } \\
\text { outcome }\end{array}$ & \multicolumn{2}{c|}{$\begin{array}{c}\text { Unfavourable } \\
\text { outcome }\end{array}$} & \multicolumn{2}{c|}{ Total (\%) } \\
& & $\mathrm{N}$ & $\%$ & $\mathrm{~N}$ & $\%$ & $\mathrm{~N}$ & $\%$ \\
Gestational Age & $<28$ weeks & 4 & 100 & 0 & 0 & 4 & 100 \\
of the study & $28-30$ weeks & 18 & 81.8 & 4 & 18.2 & 22 & 100 \\
participants & $30-32$ weeks & 16 & 88.9 & 2 & 11 & 18 & 100 \\
& $32-34 \mathrm{weeks}$ & 16 & 100 & 0 & 0 & 16 & 100 \\
Birth weight & $<1.5 \mathrm{~kg}$ & 42 & 87.5 & 6 & 12.5 & 48 & 100 \\
Involvement in & $>1.5 \mathrm{~kg}$ & 12 & 100 & 0 & 0 & 12 & \\
clock hours & $>6$ hours & 14 & 93.3 & 1 & 6.7 & 15 & 100 \\
Stage of ROP & Stage 1 or 2 & 40 & 88.8 & 5 & 11 & 45 & \\
& Stage 3 & 24 & 83.7 & 2 & 6.3 & 32 & \multirow{7}{*}{100} \\
\hline Table 4. Distribution of structural outcome of retina with gestational \\
age, Birth weight and involvement in clock hours (n-60) \\
\hline
\end{tabular}

Among the 6 eyes with unfavorable outcome, 4 were of gestational age less than30weeks but there was no statistically significant association. Among the studied infants unfavourable outcome occurred in those with birth weight less than $1.5 \mathrm{~kg}$. With low birth weight the risk for unfavourable structural outcome is high. Out of the 6 eyes with unfavourable structural outcome, 5 had more than 6 clock hours of involvement.Among the 6 eyes with unfavorable outcome, 4 had stage 3 ROP. Unfavourable structural outcome increases with Stage of ROP.

\section{Functional outcome Measures}

The functional outcome was assessed in terms of visual behavior by assessing the fixation of light, whether child is following light and by preferential looking pattern. The refractive errors were assessed by performing retinoscopy. Other sequels noted were presence of strabismus or nystagmus.

\begin{tabular}{|ccc|}
\hline Visual behaviour & Frequency & Percentage \\
\hline Favourable & 58 & $96.7 \%$ \\
Unfavourable & 2 & $3.3 \%$ \\
Total & $\mathbf{6 0}$ & $\mathbf{1 0 0}$ \\
\hline Table 5. Distribution of functional outcome among the study \\
& participants (n-60) & \\
\hline
\end{tabular}

Out of the 30 babies only 1 had an unfavourable visual behavior.The baby was not fixing and following light. The baby had global developmental delay and the abnormal visual behavior could be attributed to that since the fundal examination showed a favourable outcome.

\section{Refractive Errors}

Children are hypermetropic in the first 3years of life. In full term infants,over the first few years they undergo a process of emmetropisation. But this is disrupted in premature babies and leads to development of refractive errors. Refractive outcome was assessed after cycloplegic retinoscopy. In our study refractive errors have been classified as follows (interms of the spherical equivalent calculated from the retinoscopy):Hypermetropia: $>4 \mathrm{D}$ (Diopters), Emmetropia:04D, low myopia 0-3D, moderate myopia -3D to $-6 \mathrm{D}$, High myopia :> -6D.

\begin{tabular}{|cccc|}
\hline & Category & Frequency & Percentage (\%) \\
\hline & High myopia & 1 & $1.7 \%$ \\
Refractive error at & Moderatemyopia & 8 & $13.3 \%$ \\
6 months & Low myopia & 24 & $40.0 \%$ \\
& Normal & 17 & $28.3 \%$ \\
& Hypermetropia & 10 & $16.7 \%$ \\
& Total & 60 & $100.0 \%$ \\
Refractive error at & High myopia & 2 & 3.3 \\
10months & Low myopia & 12 & 20.0 \\
& Normal & 15 & 38.3 \\
& Hypermetropia & 8 & 25.0 \\
& Total & $\mathbf{6 0}$ & 13.3 \\
\hline Table 6. Distribution of Refractive error of study participants at 6 and \\
\cline { 2 - 3 } & 10 months (n-60) \\
\hline
\end{tabular}

At 6 months, myopia was the predominant refractive error in the studied group accounting for about 55\%.Among the study group $16.7 \%$ had hypermetropia. The mean spherical equivalent was $0.23 \mathrm{D}$ (SD 2.92D). The spherical equivalent ranged from-6.5 D to $+5.0 \mathrm{D}$ at 6 months. $28.3 \%$ had a normal refractive status. 
Myopia remained as the predominant refractive error at 10 months of age. The incidence and degree of myopia increased as age advanced. About $61 \%$ of the babies had myopia at10 months of age. $25 \%$ had normal refraction. The mean spherical equivalent at 10 months was $-0.53 \mathrm{D}$ (SD: 3.24 D; range: $-8.0 \mathrm{D}$ to $+5.0 \mathrm{D}$ ).

The incidence of myopia increased from $55 \%$ at 6 months to $61.6 \%$ at 10 months. Those with lower degrees of myopia showed a shift to medium to high myopia as age advances.

There is an increase in those with high and medium myopia while the number of hypermetropes was reduced from $16.6 \%$ to $13.3 \%$. Infants with a normal refraction were reduced from $28.3 \%$ to $25 \%$.

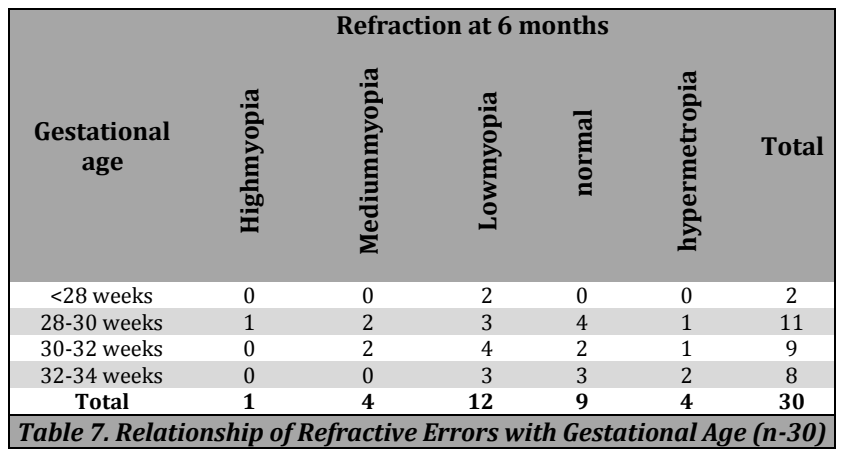

The incidence of refractive error was high in low gestational age group. All babies with gestational age less than 28 weeks had low myopia. As the gestational age increases the number of babies with myopia is decreasing. In 28-30 week age group $54.1 \%$ had myopia while it was reduced to $28 \%$ in $32-34$ age groups. Since myopia was the predominant refractive error it was further analyzed:

\begin{tabular}{|c|c|c|c|c|c|c|c|c|c|}
\hline \multirow{3}{*}{ Factors } & & \multicolumn{4}{|c|}{ Refraction at 6 months } & \multirow{2}{*}{\multicolumn{2}{|c|}{ Total }} & \multirow{3}{*}{$\chi^{2}$} & \multirow{3}{*}{$\begin{array}{c}\mathbf{P} \\
\text { value }\end{array}$} \\
\hline & Category & \multicolumn{2}{|c|}{ Myopia } & \multicolumn{2}{|c|}{ No Myopia } & & & & \\
\hline & & $\mathrm{N}$ & $\%$ & N & $\%$ & $\mathbf{N}$ & $\%$ & & \\
\hline Gestational & l $<30$ week & 15 & 577 & 11 & 42.3 & 26 & \multirow[b]{2}{*}{100} & \multirow{2}{*}{0.134} & \multirow{2}{*}{0.714} \\
\hline & $>30$ & 18 & & & & & & & \\
\hline Birt & $<1$ & 31 & & & & & \multirow{2}{*}{100} & \multirow{2}{*}{8.906} & \multirow{2}{*}{0.003} \\
\hline weight & $>1.5 \mathrm{~kg}$ & 2 & 1 & 10 & 83.3 & 12 & & & \\
\hline Age at & $<36$ week & 20 & 58.8 & 14 & 41.2 & 34 & \multirow{2}{*}{100} & \multirow{2}{*}{0.793} & \multirow{2}{*}{0.373} \\
\hline diagnosis & $>36$ week & 13 & 50 & 13 & 50 & 26 & & & \\
\hline
\end{tabular}

The incidence of myopia was high among the low gestational age group. That is as the prematurity increases the chance for myopia increases. $57.7 \%$ of infants with gestational age less than 30 weeks had myopia. It was reduced to $52.9 \%$ in more than 32 weeks age group. However the association was not statistically significant.

The incidence of myopia was $64 \%$ in less than $1.5 \mathrm{~kg}$ birth weight group while this was only $16.7 \%$ in above $1.5 \mathrm{~kg}$ birth weight group.That is low birth weight increases the risk of myopia. The association was found to be statistically significant. Early development of ROP is associated with high risk of developing myopia. $61 \%$ of infants who were diagnosed to have ROP before 36 weeks of PCA had myopia while the incidence was $50 \%$ in 34 weeks and above.60\% of the study participants were found to have astigmatism at 6 and10 months. That is, there was no worsening of astigmatism as age advances.

\section{DISCUSSION}

The study population had male female ratio 1.1.No definite gender predilection was noted.

\section{Risk Factors}

Gestationalage

The mean gestational age of participants was 30.19 weeks ( \pm 1.78 weeks.). This is comparable to a study by DeekshaKatoch, Gaurav Sanghietal in India8 (28.99 weeks) and much higher than the ETROP study cohort which had an average gestational age of 25 weeks. $^{7}$

\section{Birth Weight}

The average birth weight of infants in the present study was $1240 \mathrm{gm}$ which is much higher than ETROP cohort (705g).This is comparable to $1120 \mathrm{~g}$ in the study by Deeksha Katoch, Gaurav Sanghi et al. The rationale for difference in birth weight in Indian scenario is based on the findings by Freider et al ROP is seen in larger babies. ${ }^{9}$ Infants with low birth weight had more chance of unfavorable structural outcome and refractive errors at follow up.

\section{Disease Characteristics}

Out of the 62 eyes, $42 \%$ developed any form of ROP at a postconceptional age of 32-34 weeks. Most of these infants underwent laser treatment around 36-38 weeks (42\%).That is $42 \%$ of infants developed threshold or pre threshold type I at PCA of 36-38 weeks. This finding is comparable to CRYO ROP study ${ }^{10}$ in which the mean age of development of threshold disease is 36.9 weeks 37 weeks in ETROP study. ${ }^{7}$ Early development of ROP was associated with an increased incidence of myopia.

In our study all babies had ROP in both eyes and it was symmetrical. Out of the 62 eyes $48 \%$ had stage 2 and stage 3 disease each. Stage 3 disease was more associated with an unfavorable structural outcome.(4 out of 6 eyes with unfavorable outcome had stage 3 disease. 83.9\% eyes had zone 2 ROP in the present study and $72.5 \%$ had more than 6 clock hours of involvement. Of the 6 eyes with unfavorable structural outcome 5 were having more than 6 clock hours of involvement.

\section{Structural and Functional outcome}

Out of the 60 treated eyes, $90 \%$ had a favorable structural outcome at 3, 6 and 10 months. That is the incidence of unfavorable structural outcome is $10 \%$. This is comparable to the ETROP study where the incidence of unfavorable structural outcome is $9.1 \%$. In our study all the 6 eyes with unfavorable outcome had macular drag. Although peripheral retinal changes were not seen in our study population the risk for the same persists and hence the need for follow up examinations.

All infants with unfavourable outcome belonged to less than $1.5 \mathrm{~kg}$ birth weight group and low birth weight is a statistically significant predictor of unfavorable structural outcome. Only 2 out of 30 infants in the present study had strabismus $(6.6 \%)$ and both had esotropia. This is lower compared to ETROP study which reported strabismus in 
$22.8 \%$. This low incidence of strabismus may be attributed to short follow up time and bilateral symmetric presentation. Only 1 baby ( 2 eyes) out of the 30 had an unfavorable visual outcome and had global developmental delay and the abnormal visual behavior could be attributed to this.

Myopia was the predominant refractive error in the study group comprising around $55 \%$ at 6 months and $61 \%$ at 10 months. The ETROP study had also reported that $80 \%$ of threshold ROP infants and $65 \%$ of treated high risk prethreshold infants had myopia. Low birth weight and prematurity contributes to myopia in infancy. In addition to this the incidence of myopia rises in infants with ROP as compared to infants without ROP of same birth weight. Recent studies showed that laser treatment also contributes to myopia in ROP infants. ${ }^{11,12}$ In the present study statistically significant association was found between myopia and low birth weight. The mean spherical equivalent of the study population was $0.23 \mathrm{D}$ at 6 months and -0.53 at 10 months. That is there is a myopic shift as age advances. This emphasizes the importance of strict follow up in treated ROP infants and also the need for appropriate correction.Also theincidence of astigmatism was very high in the study group (60\%).

This study shows that timely early treatment can prevent structural sequel in ROP infants and excellent results comparable to HHDC can be obtained. Also this study stresses that the treatment of ROP must not stop with laser treatment but the need for follow up of these children by treating Ophthalmologistfurther to manage the forthcoming sequel of refractive errors and others like retinal thinning, retinal hole and detachment.

\section{CONCLUSIONS}

The incidence of favorable structural outcome in our study was $90 \%$.Low gestational age and low birth weight were associated with an unfavourable structural outcome. There isa high prevalence of refractive errors in our study group accounting for about $71.5 \%$ at 6 months. There fractive errors increased at 10 month follow up to $77 \%$. Among there fractive errors, myopia predominates accounting 55\% at 6 months and $61 \%$ at 10 months. The prevalence of myopia was significantly affected by low birth weight. There was a high prevalence of a stigmatism in our study group. Low birth weight, prematurity, ROP and laser contribute to the presence of refractive errors. The incidence of strabismus was 6.6\%in our study group.

Data sharing statement provided by the authors is available with the full text of this article at jemds.com.

Financial or other competing interests: None.
Disclosure forms provided by the authors are available with the full text of this article at jemds.com.

\section{REFERENCES}

[1] Terry TL. Fibroblastic over growth of persistent tunica vasculosa lentis in infants born prematurely: II. Report of cases-clinical aspects. Trans Am Ophthalmol Soc 1942;40:262-84.

[2] Blindness in world. Surv Ophthalmology 2000;45(Suppl 1):S21-31.

[3] Gopal L, Sharma T, Ramachandran S, et al. Retinopathy of prematurity: a study. Indian J Ophthalmology 1995;43(2):59-61.

[4] Patz A, Hoeck LE, Cruz ED. Studies on the effect of high oxygen administration in retrolental fibroplasia. I. Nursery observations. Am J Ophthalmol 1952;35(9):1248-53.

[5] Ling CS, Fleck BW, Wright E, et al. Diode laser treatment for retinopathy of prematurity: structural and functional outcome. Br J Ophthalmology 1995;79(7):637-41.

[6] Laws F, Laws D, Clark D. Cryotherapy and laser treatment for acute retinopathy of prematurity: refractive outcomes a longitudinal study. $\mathrm{Br}$ J Ophthalmology 1997;81(1):12-5.

[7] Early Treatment for Retinopathy of Prematurity Cooperative Group. Revised indications for the treatment of retinopathy of prematurity: results of the early treatment for retinopathy of prematurity randomized trial. Arch Ophthalmol 2003;121(12):1684-94.

[8] Katoch D, Sanghi G, Dogra MR, et al. Structural sequelae and refractive outcome 1 year after laser treatment for type 1 prethreshold retinopathy of prematurity in Asian Indian eyes. Indian J Ophthalmol 2011;59(6):423-6.

[9] Fielder AR, Shaw DE, Robinson J, et al. Natural history of retinopathy of prematurity: a prospective study. Eye (Lond) 1992;6(Pt 3):233-42.

[10] Quinn GE, Dobson V, Kivlin J, et al. Prevalence of myopia between 3 months and $51 / 2$ years in preterm infants with and without retinopathy of prematurity. Cryotherapy for Retinopathy of Prematurity Cooperative Group. Ophthalmology 1998;105(7):1292-300.

[11] Dhawan A, Dogra M, Vinekar A, et al. Structural sequelae and refractive outcome after successful laser treatment for threshold retinopathy of prematurity. J Pediatr Ophthalmol Strabismus 2008;45(6):356-61.

[12] McLoone EM, O'Keefe M, McLoone SF, et al. Long-term refractive and biometric outcomes following diode laser therapy for retinopathy of prematurity. J AAPOS 2006;10(5):454-9. 